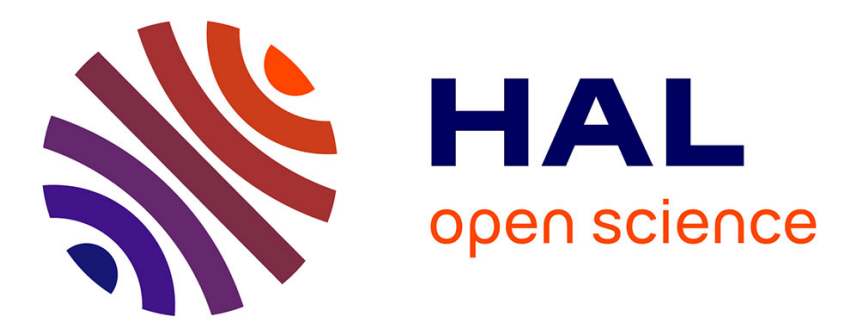

\title{
Optical generation and detection of gigahertz shear acoustic waves in solids assisted by a metallic diffraction grating
}

Osamu Matsuda, Kandai Tsutsui, Gwenäelle G. Vaudel, Thomas Pezeril, Kentaro Fujita, Vitali Goussev

\section{To cite this version:}

Osamu Matsuda, Kandai Tsutsui, Gwenäelle G. Vaudel, Thomas Pezeril, Kentaro Fujita, et al.. Optical generation and detection of gigahertz shear acoustic waves in solids assisted by a metallic diffraction grating. Physical Review B, 2020, 101 (22), 10.1103/PhysRevB.101.224307 . hal-03007539

\section{HAL Id: hal-03007539 \\ https://hal.science/hal-03007539}

Submitted on 8 Oct 2021

HAL is a multi-disciplinary open access archive for the deposit and dissemination of scientific research documents, whether they are published or not. The documents may come from teaching and research institutions in France or abroad, or from public or private research centers.
L'archive ouverte pluridisciplinaire HAL, est destinée au dépôt et à la diffusion de documents scientifiques de niveau recherche, publiés ou non, émanant des établissements d'enseignement et de recherche français ou étrangers, des laboratoires publics ou privés. 


\begin{tabular}{|c|l|}
\hline Title & Optical generation and detection of gigahertz shear acoustic waves in solids assisted by a metallic diffraction grating \\
\hline Author(s) & Matsuda, O samu; Tsutsui, Kandai; V audel, Gwenaelle; Pezeril, Thomas; Fujita, Kentaro; Gusev, V italyi \\
\hline Citation & $\begin{array}{l}\text { Physical Review B, 101(22), 224307 } \\
\text { https://doi.org/10.1103/PhysRevB.101.224307 }\end{array}$ \\
\hline Issue Date & 2020-06-22 \\
\hline Doc URL & http://hdl.handle.net/2115//8973 \\
\hline Rights & Copyright (2020) by The A merican Physical Society. \\
\hline Type & article \\
\hline File Information & PhysRevB.101.224307.pdf \\
\hline
\end{tabular}

Instructions for use 


\title{
Optical generation and detection of gigahertz shear acoustic waves in solids assisted by a metallic diffraction grating
}

\author{
Osamu Matsuda $\odot,{ }^{1, *}$ Kandai Tsutsui, ${ }^{1}$ Gwenaëlle Vaudel $\odot,{ }^{2}$ Thomas Pezeril,${ }^{2}$ Kentaro Fujita $\odot,{ }^{1}$ and Vitalyi Gusev $\odot^{3, \dagger}$ \\ ${ }^{1}$ Division of Applied Physics, Faculty of Engineering, Hokkaido University, Sapporo, Hokkaido 060-8628, Japan \\ ${ }^{2}$ Institut des Molécules et Matériaux du Mans, UMR 6283 CNRS, Le Mans Université, Avenue Olivier Messiaen, 72085 Le Mans, France \\ ${ }^{3}$ Laboratoire d'Acoustique de l'Université du Mans, UMR 6613 CNRS, Le Mans Université, Avenue Olivier Messiaen, 72085 Le Mans, France
}

(Received 11 April 2020; revised manuscript received 30 May 2020; accepted 2 June 2020;

published 22 June 2020)

\begin{abstract}
Absorption of ultrashort laser pulses in a metallic grating deposited on a transparent sample launches in the material both compression/dilatation (longitudinal) and shear coherent acoustic pulses in directions of different orders of acoustic diffraction. The propagation of the emitted acoustic pulses can be monitored by measuring the variation of the optical reflectivity of the time-delayed ultrashort probe laser pulses. The direction of probe light incidence and its polarization relative to the sample surface as well as the orientation of the metallic grating should be specifically chosen for efficient Brillouin scattering of the probe light from shear phonons propagating in the elastically isotropic materials. As theoretically predicted, the obtained experimental data contain multiple frequency components which are due to a variety of possible Brillouin scattering angles for both shear and compression/dilatation coherent acoustic waves. All these different frequency components are explained through multiplexing the propagation directions of probe light and coherent sound by the metallic diffraction grating. Our experimental scheme of time-domain Brillouin scattering with metallic gratings operating in reflection mode provides access to monitoring the shear acoustic waves launched in the direction of the first diffraction order by backward Brillouin scattering process. Applications include simultaneous determination of several different acoustic mode velocities and optical refractive index and, potentially, measurements of the acoustic dispersion of samples with a single direction of possible optical access.
\end{abstract}

DOI: 10.1103/PhysRevB.101.224307

\section{INTRODUCTION}

Absorption of ultrashort light pulses with picosecond temporal width in a medium may generate therein coherent acoustic waves in the gigahertz $(\mathrm{GHz})$ frequency range. The propagation of the acoustic waves is accompanied by the modulation of optical reflectivity via the photoelastic effect and thus can be monitored by the time-delayed light pulses [1]. The technique is often called picosecond laser ultrasonics and is utilized to investigate the elastic properties of the medium; the structure parameters such as the thickness of thin films, the elasto-optic properties, etc. [1-7]; and imaging inside transparent inhomogeneous media with suboptical and even nanometric depth spatial resolution [8]. In most cases, only the longitudinal acoustic waves have been used for these measurements because of the symmetry of the samples and measurements. It is, however, highly desirable to generate and detect shear acoustic waves in addition to longitudinal acoustic waves in order to get the full mechanical information of the elasticity of the medium. Such attempts require lower symmetry in the medium and/or excitation configurations: for example, the use of an anisotropic medium [9-19] or the use of acoustic waves propagating along the direction non-normal to the surface $[20,21]$. It has also been suggested

\footnotetext{
*omatsuda@eng.hokudai.ac.jp

†vitali.goussev@univ-lemans.fr
}

that optically induced gratings formed by the illumination of the sample surface with two nonparallel light beams may generate shear acoustic waves [22,23]. In conjunction with this generic concept, we previously conducted time-domain Brillouin measurements in fused silica samples with metallic grating photoacoustic transducers and showed that the longitudinal acoustic waves propagating along multiple directions are observed simultaneously, giving rise to several distinct Brillouin frequencies [24]. Even though shear acoustic waves are expected to be excited in these grating structures, we did not succeeded in revealing them [24].

In this paper, we clarify the necessary condition for the optical detection of shear acoustic waves in time-resolved Brillouin measurements for transparent samples with metallic grating photoacoustic transducers. Our experiments reveal the unambiguous detection of shear acoustic waves in addition to longitudinal acoustic waves. We then evaluate the shear and longitudinal sound velocities, as well as the refractive index of fused silica, as a demonstration of the capability of this method.

\section{THEORY}

The generation and detection of longitudinal acoustic waves in a transparent isotropic medium using metallic gratings were described in detail elsewhere [24,25]. Absorption of ultrashort light pulses at the metallic grating may generate longitudinal acoustic waves propagating not only normally 


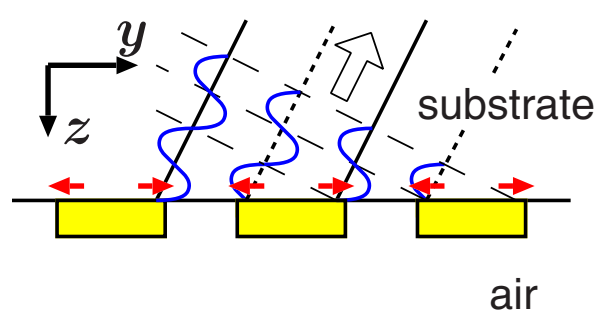

FIG. 1. Schematic diagram of the generation of shear acoustic waves by the optical excitation of metallic gratings. The upper half-space is an isotropic medium. The metallic grating rods are formed on its surface: their cross sections are shown as the yellow rectangles, and their expansion along the $y$ direction is indicated by red arrows. The blue waves schematically show the displacement of the generated shear acoustic waves propagating along the first-order diffraction (white arrow).

to the plane of the gratings but also in all directions corresponding to the possible nonzeroth diffraction orders of the acoustic waves induced by this grating, thus multiplexing the propagation directions of the generated acoustic waves in the sample. The propagation of the generated acoustic waves is optically detected by the time-delayed light pulses that measure the transient reflectivity change of the sample, showing the so-called Brillouin oscillations. In the measurement process, the probe light scattered by the acoustic field and the probe light reflected or diffracted by the grating structure interfere on the photodetector. The grating can diffract the probe light before and/or after its scattering by the acoustic field in both transmission and reflection configurations, thus multiplexing the propagation directions of the probe laser pulses inside the sample. These multiple combinations of sound and light propagation directions yield the observation of several different oscillation frequencies in both backward and forward Brillouin scattering simultaneously [24].

In the present paper, we focus on the generation and detection by metallic gratings of shear acoustic waves. Let us suppose that an ultrashort light pulse is absorbed by a metallic grating formed on a transparent isotropic medium. Figure 1 shows the cross section of the sample. The $x$ axis is taken along the grating rods, the $y$ axis along the grating repetition, and the $z$ axis is perpendicular to the surface on which the grating structure is formed. The absorption of the light pulse in the metallic rods sets up an instantaneous temperature rise and thus the thermal stress distribution. Then the rods tend to expand laterally, i.e., in the $y$ direction, in addition to the $z$ direction: thus, the stress component $\sigma_{y z}$ is nonzero at the interface between the rod and the substrate, especially near the edge of the rods.

This mechanism of shear acoustic wave generation can be described in detail as follows. The heating of the metallic rods by laser radiation and heat transfer from the rods to the transparent substrate induce a temperature rise that gives rise to isotropic thermoelastic stresses in both of these media exhibiting isotropic thermal expansion. The isotropic thermoelastic stresses generate only compression/dilatation (longitudinal) acoustic waves [12,13,21]. However, both in the elastically isotropic substrate $\left(\mathrm{SiO}_{2}\right.$ in our case) and in the metallic grating (polycrystalline $\mathrm{Al}$ in our case), the compression/dilatation waves obliquely incident on the surfaces and interfaces get partially mode converted into shear acoustic waves. Thus, in our experiments, the shear acoustic waves are emitted into the substrate, in general, through three different channels: the mode conversion in transmission of the compression/dilatation waves incident on the $\mathrm{SiO}_{2} / \mathrm{Al}$ interface from the $\mathrm{Al}$ side, the mode conversion in the reflection of the compression/dilatation waves incident on the $\mathrm{SiO}_{2} / \mathrm{Al}$ interface from the $\mathrm{SiO}_{2}$ side [26], and the transmission through the $\mathrm{SiO}_{2} / \mathrm{Al}$ interface of the shear acoustic waves incident from the Al side. In the third channel, the shear acoustic waves are most efficiently excited in the Al rods by the mode conversion of the compression/dilatation waves photogenerated in $\mathrm{Al}$ rods when they are incident on the mechanically free surfaces of the Al rods. Since the polarity of $\sigma_{y z}$ alternates between the right and left edges in each individual rod, $\sigma_{y z}$ does not generate plane homogeneous shear acoustic waves, which could propagate perpendicularly to the grating surface. In other words, the considered grating does not break in average left-right symmetry of the surface. Thus, the generation of the plane shear acoustic waves propagating normally to the surface is forbidden by symmetry. It is, however, not excluded to generate plane inhomogeneous shear acoustic waves (acoustic gratings) [27]. Each of the plane inhomogeneous shear waves is a superposition of two plane shear acoustic waves emitted by the grating in the directions of two opposite diffraction orders. Thus, the amplitude modulation in the plane inhomogeneous shear waves results from the propagation of two plane homogeneous waves at an angle from each other. Because of the symmetry of the system, the displacement polarization of the generated shear acoustic waves is in the $y z$ plane.

As for the detection, we first concentrate on an elementary process of the light scattering by the shear acoustic waves with arbitrary polarization, excluding the light reflection or diffraction by the metallic grating before and/or after the scattering by the coherent shear acoustic wave. The momentum conservation in the scattering process is described as

$$
\mathbf{k}_{s}=\mathbf{k}_{i} \pm \mathbf{k}_{B},
$$

where $\mathbf{k}_{i}$ is the wave vector of the incident light (incident state of a photon), $\mathbf{k}_{s}$ is the wave vector of the light scattered by the acoustic waves (scattered state of a photon), and $\mathbf{k}_{B}$ is the wave vector of the acoustic phonon involved in the scattering process. The plus sign corresponds to phonon annihilation, whereas the minus sign corresponds to phonon creation. For the analysis of Eq. (1), we take the $x_{3}$ axis along $\mathbf{k}_{B}$ and the $x_{1}$ axis in the plane defined by $\mathbf{k}_{s}$ and $\mathbf{k}_{i}$ (plane of incidence), as shown in Fig. 2. The light may take the $p$ polarization in which the electric field is in the $x_{1}-x_{3}$ plane or the $s$ polarization in which the electric field is along the $x_{2}$ axis (perpendicular to the $x_{1}-x_{3}$ plane). The shear acoustic wave may have its displacement polarization along the $x_{1}$ or $x_{2}$ axis corresponding to nonzero strain component $\eta_{13}$ or $\eta_{23}$, respectively.

The light scattering by the acoustic wave is, in fact, the light emission of the electric polarization induced by the incident light and the acoustic wave. Considering the photoelastic effect in the isotropic medium, the electric polarization 
(a)

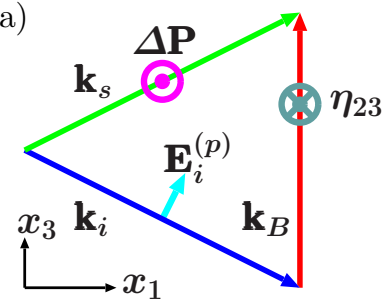

(c)

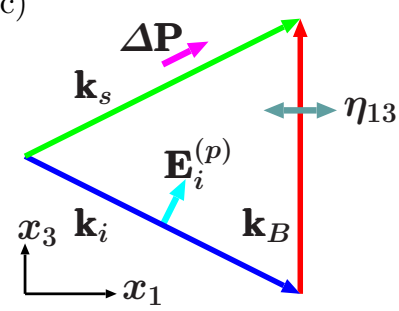

(b)

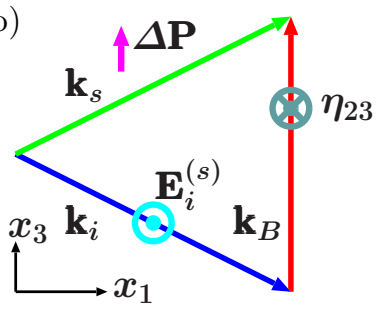

(d)

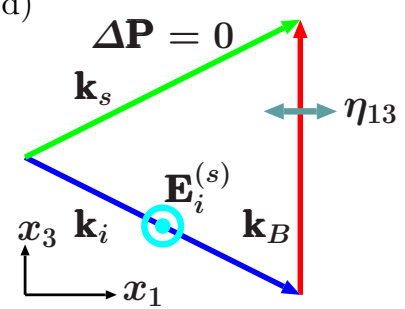

FIG. 2. Momentum conservation and induced electric polarization $\Delta \mathbf{P}$ in the scattering process of the incident light $\left(\mathbf{k}_{i}\right)$ into the scattered light $\left(\mathbf{k}_{s}\right)$ by the acoustic waves $\left(\mathbf{k}_{B}\right)$. (a) The $p$ incident light and the $x_{2}$-polarized shear waves. (b) The $s$ incident light and the $x_{2}$-polarized shear waves. (c) The $p$ incident light and the $x_{1}$-polarized shear waves. (d) The $s$ incident light and the $x_{1}$-polarized shear waves.

$\Delta \mathbf{P}$ induced by the shear strain and the incident light is given as

$$
\Delta \mathbf{P} \propto\left(\begin{array}{ccc}
0 & 0 & P_{44} \eta_{13} \\
0 & 0 & P_{44} \eta_{23} \\
P_{44} \eta_{13} & P_{44} \eta_{23} & 0
\end{array}\right)\left(\begin{array}{l}
E_{i 1} \\
E_{i 2} \\
E_{i 3}
\end{array}\right),
$$

where $P_{44}$ is a component of the photoelastic tensor of the isotropic medium. $E_{i j}$ is the $j$ th component of the electric field $\mathbf{E}_{i}$ of the incident light. For the $p$-polarized light, $\mathbf{E}_{i}$ is directed as

$$
\mathbf{E}_{i}^{(p)} \| \frac{1}{\left|\mathbf{k}_{i}\right|}\left(\begin{array}{c}
-k_{i 3} \\
0 \\
k_{i 1}
\end{array}\right),
$$

where $k_{i j}$ is the $j$ th component of the wave vector $\mathbf{k}_{i}$ of the incident light. For the $s$-polarized light, it is directed as

$$
\mathbf{E}_{i}^{(s)} \|\left(\begin{array}{l}
0 \\
1 \\
0
\end{array}\right) .
$$

As depicted in Fig. 2, there are four possible combinations of the shear displacement polarization and the incident light polarization. The light is emitted from the induced electric polarization projected to the plane perpendicular to the scattered light wave vector $\mathbf{k}_{s}$. In the case of $p$-polarized incident light and $x_{2}$-polarized shear acoustic waves [Fig. 2(a)], the induced electric polarization is along the $x_{2}$ axis which is perpendicular to the scattered light propagation direction. This is the most suitable configuration for the shear acoustic wave detection. In case of $s$-polarized incident light and $x_{2}$-polarized shear acoustic waves [Fig. 2(b)], the induced electric polarization is along the $x_{3}$ axis. The projection of this electric polarization to the plane perpendicular to the scattered light propagation direction is nonzero unless $\mathbf{k}_{s} \| x_{3}$, and thus, shear acoustic wave detection may be possible in this configuration. In the case of $p$-polarized incident light and $x_{1}$-polarized shear acoustic waves [Fig. 2(c)], the induced electric polarization is parallel to the scattered light propagation direction, and thus, it is not possible to detect the shear acoustic waves. In the case of $s$-polarized incident light and $x_{1}$-polarized shear acoustic waves [Fig. 2(d)], there is no induced electric polarization, and thus, it is not possible to detect the shear acoustic waves. As a summary of these four configurations, the shear acoustic waves can be detected only if the plane of probe light incidence (the $x_{1}-x_{3}$ plane) is not parallel to the polarization (mechanical displacement) of the shear acoustic wave.

As seen in the generation process, the shear displacement lies in the plane perpendicular to the grating rods. Thus, the above discussion tells us that, for shear acoustic wave detection, the propagating direction of the incident light to the sample should not be chosen in the plane perpendicular to the grating rods.

\section{EXPERIMENT}

The sample is made of a fused silica substrate with a thickness of $1 \mathrm{~mm}$. By the electron beam lithography and the lift-off technique, the aluminum grating structure is formed on the surface of the substrate. The grating period $p$ is $380 \mathrm{~nm}$. The width of the aluminum rod is $190 \mathrm{~nm}$, and the thickness is $40 \mathrm{~nm}$. The total grating area is of $100 \times 100 \mu \mathrm{m}^{2}$.

A standard optical pump-probe setup is used for the measurement [24]. A Ti:sapphire mode-locked laser is used as the light source. It generates the light pulses at the central wavelength of $830 \mathrm{~nm}$, temporal width of $100 \mathrm{fs}$, and repetition frequency of $80 \mathrm{MHz}$. The $830-\mathrm{nm}$ wavelength light pulses (pump light pulses) with an energy of $0.03 \mathrm{~nJ} /$ pulse are focused to the grating region of the sample from the backside, i.e., from the side without the grating, to generate the acoustic waves. The diameter of the pump focus spot is about $50 \mu \mathrm{m}$. Part of the 830-nm wavelength light pulses is fed to a secondharmonic-generation crystal $\left(\beta-\mathrm{BaB}_{2} \mathrm{O}_{4}\right)$ for conversion into 415-nm-wavelength light pulses (probe light pulses) with an energy of $0.04 \mathrm{~nJ} /$ pulse, which are time delayed and focused on the grating region also from the backside to detect the acoustic waves. The diameter of the probe focus spot is about $20 \mu \mathrm{m}$. The transient optical reflectivity change is recorded by varying the delay time between the pump and probe light pulse arrivals to the sample.

As discussed in the previous section, in order to detect the shear acoustic waves, it is required that the plane of incidence of the probe light is not parallel to the plane perpendicular to the grating rods. To fulfill this condition, the sample is placed on a two-axis rotation stage and is rotated as described in the following.

Let us define the laboratory coordinate system $X Y Z$ and the sample coordinate system $x y z$. The $Y$ axis is along the vertical direction of the laboratory, and the $X-Z$ plane is parallel to the horizontal plane of the laboratory. The laser beam incident on the sample is along the horizontal $Z$ axis. 

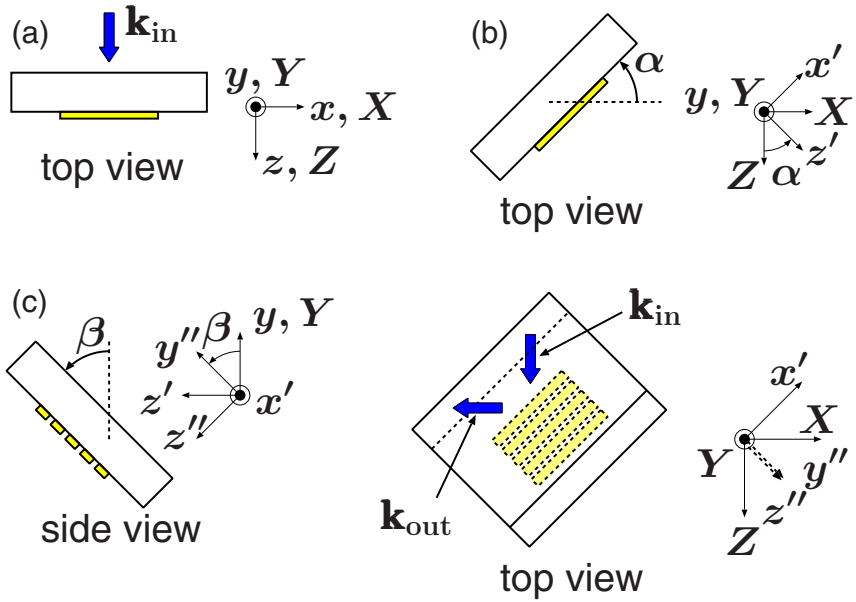

FIG. 3. Rotation of the sample. (a) Initial orientation. (b) Rotation with respect to $y$ (or $Y$ ) by an angle $\alpha$. (c) Rotation with respect to $x^{\prime}$ by an angle $\beta$ (side and top views). In the top view, $y^{\prime \prime}$ and $z^{\prime \prime}$ appear to be overlapped, but in fact, the former is directed upwards from the $X-Z$ plane, whereas the latter is directed downwards.

The $z$ axis is taken perpendicular to the sample surface facing upwards from the grating surface. The $x$ axis is along the grating rods, and the $y$ axis is along the grating periodicity, i.e., perpendicular to the rods.

First, we place the sample so that the $x y z$ and $X Y Z$ coordinate systems coincide, as shown in Fig. 3(a). The probe laser beam is incident from the back surface of the sample (without grating) along $+Z($ or $+z)$. Then the sample is rotated with respect to the $Y$ (or $y$ ) axis by an angle $\alpha$, as shown in Fig. 3(b). The second and final rotation is done with respect to the $x^{\prime}$ axis by an angle $\beta$, as shown in Fig. 3(c). This side view is seen in the direction opposite that of the $x^{\prime}$ axis. For $0<\beta<\pi / 2$, the grating surface faces downwards. For simplicity, hereafter we will refer to the rotated coordinates system $x^{\prime} y^{\prime \prime} z^{\prime \prime}$ as $x y z$.

The probe laser beam directed to the sample has the wave vector $(0,0, k)$ in the $X Y Z$ coordinates, where $k$ is the wave number of the probe light in the air. In the $x y z$ coordinates, it is expressed as

$$
\mathbf{k}_{\text {in }} \equiv\left(k_{x}, k_{y}, k_{z}\right)=k(-\sin \alpha, \cos \alpha \sin \beta, \cos \alpha \cos \beta) .
$$

The laser beam reflected by or diffracted from the sample is fed to the photodetector to observe the transient reflectivity change. The wave vector of this light in the $x y z$ coordinate system is expressed as

$$
\begin{aligned}
\mathbf{k}_{\text {out }} & =\left(k_{x}, k_{y}+m q, k_{z}^{\prime}\right), \\
k_{z}^{\prime} & =-\sqrt{k^{2}-k_{x}^{2}-\left(k_{y}+m q\right)^{2},}
\end{aligned}
$$

where $q=2 \pi / p$ is the grating wave number and $m$ is an integer determined by the choice of the light beam used for the detection: $m=0$ for the reflected light, whereas $m \neq 0$ for the light directed into the $m$ th diffraction order. For the detection in the far field, the possible $m$ should ensure the square root in Eq. (6) is real. We ignore the frequency shift of the scattered light, i.e., assume $\left|\mathbf{k}_{\text {in }}\right|=\left|\mathbf{k}_{\text {out }}\right|$ throughout this paper.

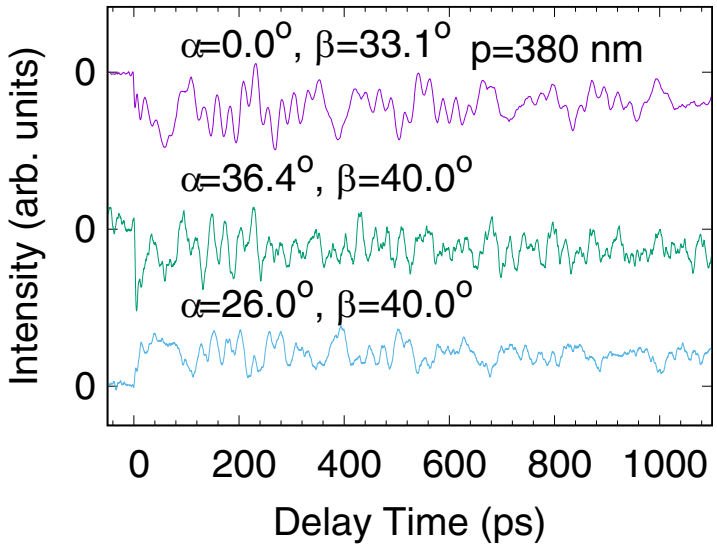

FIG. 4. Transient reflectivity changes for the sample rotations indicated on the plots.

Note that $\mathbf{k}_{\text {in }}$ and $\mathbf{k}_{\text {out }}$ are different from $\mathbf{k}_{i}$ and $\mathbf{k}_{s}$ in Eq. (1). The former describe the light state outside of the sample, whereas the latter describe the light state inside of the sample.

For the 415-nm wavelength probe light at normal incidence to the sample $\left(\alpha=\beta=0^{\circ}\right)$, the chosen grating period of $380 \mathrm{~nm}$ inhibits the diffraction outside of the sample, but it still permits its diffraction inside the sample. And it is also possible to have the diffraction outside of the sample for an obliquely incident probe at a certain range of the incident angle, as will be seen in the next section.

\section{RESULTS}

The transient reflectivity changes are measured for three different sample orientations: $[\alpha, \beta]=\left[0.0^{\circ}, 33.1^{\circ}\right]$, $\left[36.4^{\circ}, 40.0^{\circ}\right]$, and $\left[26.0^{\circ}, 40.0^{\circ}\right]$. The orientation $\left[0.0^{\circ}, 33.1^{\circ}\right]$ is chosen so that the direction of the scattered light outside of the sample in one of the first diffraction orders $(m=-1)$ is opposite the direction of the incident probe beam. This peculiarity happens when $k_{\text {out } y}=k_{y}+m q=-k_{y}$, i.e., when $2 k_{y}=2 k \sin \beta=-m q$, for $\beta \simeq 33.1^{\circ}$ in our experiment. The orientation $\left[36.4^{\circ}, 40.0^{\circ}\right]$ is chosen so that the direction of the $m=-1$ probe light outside of the sample lies in the $X-Z$ plane. The orientation $\left[26.0^{\circ}, 40.0^{\circ}\right]$ is chosen to see the effect on the time-domain Brillouin scattering signals of the variation of $\alpha$ from its previous value.

For all orientations, the light incident on the sample is linearly polarized along $Y$. The light diffracted in the first diffraction order $(m=-1)$ is detected with a photodetector for all three orientations. The polarization component along $Y$ (more precisely, the polarization component in the plane defined by the detected light beam and $Y$ axis) is chosen for the detection.

Figure 4 shows the typical transient reflectivity changes measured at three orientations. Figure 5 shows the norm of the Fourier amplitude of the transient reflectivity changes shown in Fig. 4. As explained in Sec. II, only the longitudinal peaks are expected, and the shear wave peaks are not expected in the orientation $\left[0.0^{\circ}, 33.1^{\circ}\right]$, where the propagation direction of the probe light lies in the plane perpendicular to the grating rods. In contrast, the other two orientations are expected to 


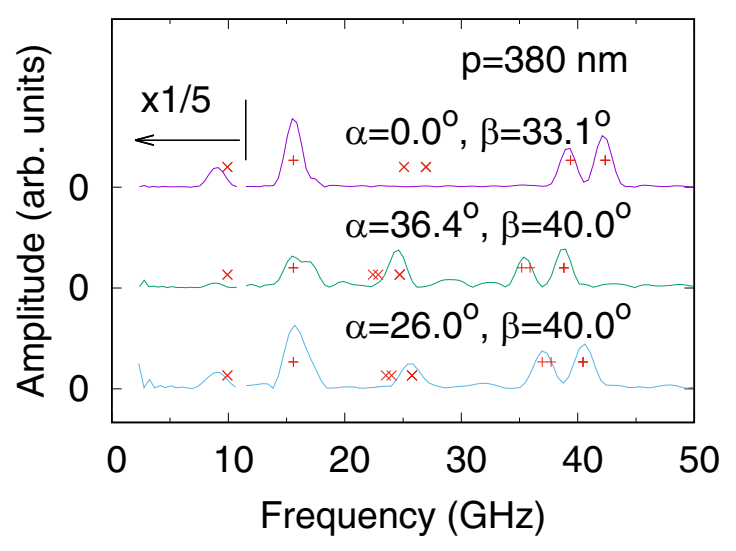

FIG. 5. Norm of Fourier amplitude of the transient reflectivity changes obtained for the sample orientations indicated on the plots. The + and $\times$ symbols indicate the calculated Brillouin frequencies for the longitudinal and shear waves, respectively. For convenience of presentation, the amplitudes in the low-lying part of the spectra $(<11 \mathrm{GHz})$ are diminished five times.

show the shear wave Brillouin frequency peaks as well as the longitudinal peaks.

The Brillouin frequency $f_{B}$ is calculated using Eq. (1), taking into account the probe light reflection or diffraction at the metal grating preceding and/or succeeding the elementary light scattering process by the acoustic waves as

$$
\begin{aligned}
f_{B}= & \frac{v}{2 \pi}\left\{\left[\left(m_{s}-m_{i}\right) q\right]^{2}+\left[d_{s} \sqrt{n^{2} k^{2}-k_{x}^{2}-\left(k_{y}+m_{s} q\right)^{2}}\right.\right. \\
& \left.\left.-d_{i} \sqrt{n^{2} k^{2}-k_{x}^{2}-\left(k_{y}+m_{i} q\right)^{2}}\right]^{2}\right\}^{1 / 2},
\end{aligned}
$$

where $v$ is the velocity of a particular acoustic mode, i.e., the velocity of the compression/dilatation, shear, or surface acoustic mode, and $m_{i}$ and $m_{s}$ are the diffraction orders for the incident and scattered light. The positive $m$ value corresponds to the diffraction towards the $+y$ direction. $d_{i}$ and $d_{s}$ take the values \pm 1 depending on the sign of the $z$ components of the wave vectors of the incident and scattered light: $d_{i, s}=1$ for the light propagating towards $+z$. The $k_{x}$ and $k_{y}$ are the ones that appeared in Eqs. (5) and (6) and are determined by the sample rotation. Because of the symmetry of the system, $k_{x}$ and $k_{y}$ are conserved also in the sample when the light traverses the back surface (without grating) of the sample. Equation (7) differs from Eq. (2) in Ref. [24] only by the additional contributions from the $k_{x}=-k \sin \alpha$ components of the light wave vectors which become nonzero when the sample is rotated by an angle $\alpha \neq 0$ to allow the detection of the shear acoustic waves.

The calculated Brillouin frequencies are marked in Fig. 5. The + and $\times$ symbols indicate the Brillouin frequencies for the longitudinal and shear waves, respectively. For this calculation, we used the longitudinal and shear sound velocities $v_{l}=5922 \mathrm{~m} / \mathrm{s}$ and $v_{t}=3772 \mathrm{~m} / \mathrm{s}$, and the refractive index at $\lambda=415 \mathrm{~nm}$ is $n=1.484$, which are all determined from the experimental data obtained here using the least-squares residual fitting. The longitudinal peaks agree very well with the experimental peaks [24]. The Brillouin frequency peaks of the shear acoustic waves are theoretically expected around
$25 \mathrm{GHz}$. In agreement with the theoretical predictions they are experimentally observed in Fig. 5 only for $\alpha \neq 0$. The experimental peak frequencies for $\alpha \neq 0$ agree well with the calculations. There are some calculated peaks which are not observed in the experiments. This will be discussed later. Note that the fitted values for $v_{l}, v_{t}$, and $n$ agree well with the literature values $v_{l}=5968 \mathrm{~m} / \mathrm{s}, v_{t}=3764 \mathrm{~m} / \mathrm{s}$, and $n=$ 1.470 at a wavelength of $415 \mathrm{~nm}$ for fused silica at room temperature [28].

It has been reported that $\mathrm{GHz}$ acoustic waves are highly sensitive to the adhesion between the film and the substrate [29-31]. The low quality of adhesion may increase the acoustic reflection at the interface and thus may increase the quality of the resonance vibrations of the rods on the substrate. On the other hand, the good adhesion quality may cause strong leakage of the resonant vibrations in the substrate and overdamped resonances if the acoustic impedance matches at the interface. In our case, the nondetectability of the resonances, which could be expected at frequencies exceeding $10 \mathrm{GHz}$, is a sign of reasonably good adhesion because the impedance matching of $\mathrm{Al}$ to glass is very good. The observations of the resonant oscillations of the gold rods forming the grating on the glass surface were reported earlier $[24,25]$. This was possible because the differences in impedances of gold and glass are significant, leading to reflection coefficients of the longitudinal and shear waves incident from the rod on the substrate exceeding $66 \%$ and $48 \%$, respectively, and the resonances are of a rather high quality. In the case of $\mathrm{Al}$ rods, these reflections coefficients are below $12 \%$ and $1.3 \%$, respectively, suggesting that the resonances of $\mathrm{Al}$ rods on the glass substrate are strongly damped (leaky). It should also be noted that the quality of the adhesion may affect the intensity of the Brillouin oscillations observed in the substrate but not their frequencies, which depend solely on the sound velocity, the refractive index of the substrate medium, and the period of grating.

\section{DISCUSSION}

The calculated Brillouin frequencies and the observed peak frequencies, as well as the indices $\left(m_{i}, d_{i}\right)$ and $\left(m_{s}, d_{s}\right)$, which identify the scattering process for a given compression/dilatation or shear acoustic mode, are summarized in Table I. The agreement between the calculated frequencies and the experimental results is reasonably good for the longitudinal (compression/dilatation) acoustic waves as reported before.

Figure 6 shows the photon and phonon wave vector diagrams described by Eq. (1) for the light scattering identified by $\left(m_{i}, d_{i}\right) \rightarrow\left(m_{s}, d_{s}\right)$ in the configuration $[\alpha, \beta]=$ $\left[0.0^{\circ}, 33.1^{\circ}\right]$. The blue arrows indicate the wave vector of incident light, the green arrows indicate that of scattered light, and the red arrows indicate that of absorbed acoustic waves. The top left one is the backward scattering by the acoustic waves without the reflection or diffraction by the metallic grating. The probe light incident on the sample corresponds to $\left(m_{i}, d_{i}\right)=(0,1)$, and the probe light diffracted from the sample corresponds to $\left(m_{s}, d_{s}\right)=(-1,-1)$. The second diagram in the upper row does not involve acoustic phonons, while the third one corresponds to the forward scattering by 
TABLE I. Calculated (Calc.) and experimental (Expt.) frequencies of acoustic waves involved in each Brillouin scattering configuration, in which $m$ and $d$ in the notation $(m, d)$ give the diffraction order and the sign of the $z$ component of the light wave vector, respectively. Frequency values in parentheses indicate that the corresponding peaks are not distinguishable. A dash (-) indicates that the corresponding peak is not observed. The rows with no values indicate that the mentioned configuration is degenerate to the one with values below. The top part shows the longitudinal acoustic waves, and the bottom part shows the shear acoustic waves. Frequency is in $\mathrm{GHz}$.

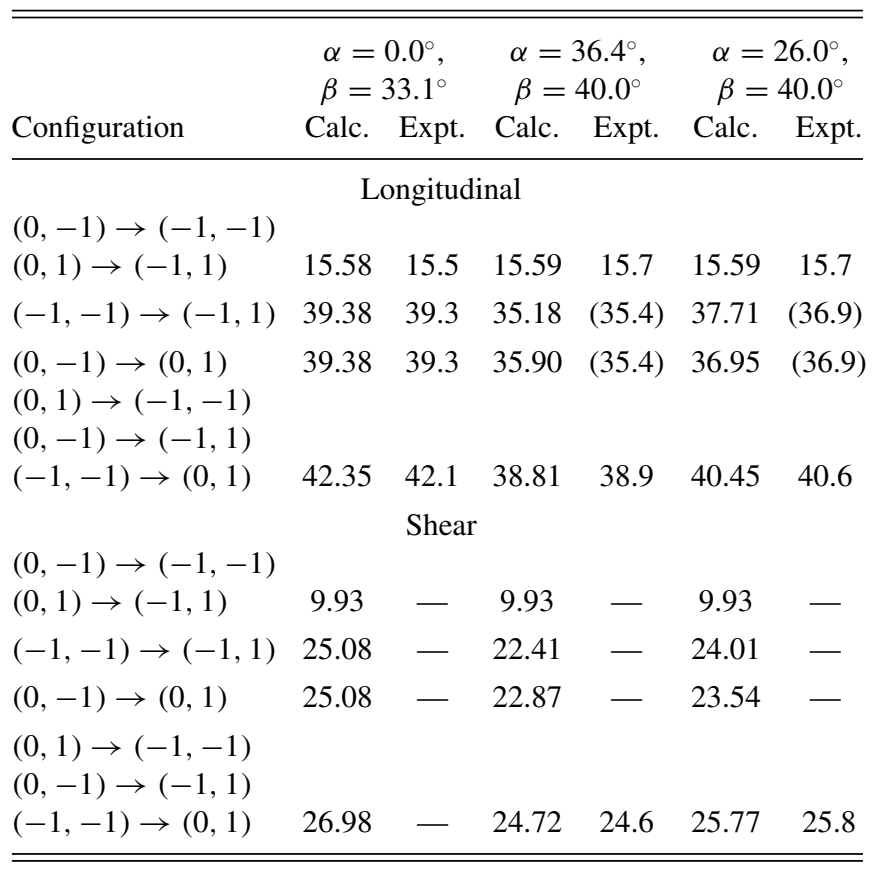

the acoustic wave with the succeeding reflection $(-1,1) \rightarrow$ $(-1,-1)$ by the metallic grating. The second diagram in the left column corresponds to the forward scattering by the acoustic wave with the preceding reflection $(0,1) \rightarrow(0,-1)$ by the metallic grating, while the third one does not involve phonons. The bottom right four configurations correspond to the backward scattering of light by the acoustic wave with both preceding and succeeding reflection and/or diffraction by the metallic grating. Because of the chosen setup, no light comes in from $\left(m_{i}, d_{i}\right)=(-1,1)$, and no light is detected at $\left(m_{s}, d_{s}\right)=(0,-1)$; thus, these states are not included in Fig. 6 . The degeneracy of the scattering processes $(0,-1) \rightarrow$ $(0,1)$ and $(-1,-1) \rightarrow(-1,1)$ is clearly shown, although this degeneracy is a consequence of the choice of the equality $k_{x}=q / 2$. This degeneracy is resolved in other configurations, $[\alpha, \beta]=\left[36.4^{\circ}, 40.0^{\circ}\right]$ and $\left[26.0^{\circ}, 40.0^{\circ}\right]$, where $k_{x} \neq q / 2$.

For the shear acoustic waves, the experimental peaks around $25 \mathrm{GHz}$ observed only in two measurements at nonzero $\alpha$ angles agree well with the calculated frequencies for $(0,1) \rightarrow(-1,-1),(0,-1) \rightarrow(-1,1)$, and $(-1,-1) \rightarrow$ $(0,1)$. The listed processes are all related to the backward scattering Brillouin processes (as the symbol $d$ changes sign in the process) by the shear phonons propagating along one of the directions of the first-order diffraction (as $\left|m_{i}-m_{s}\right|=$ $1)$. For $d_{i}=1$ the incident light is directly scattered by the acoustic grating, whereas for $d_{i}=-1$ the incident light is first reflected or diffracted by the metallic grating, then scattered

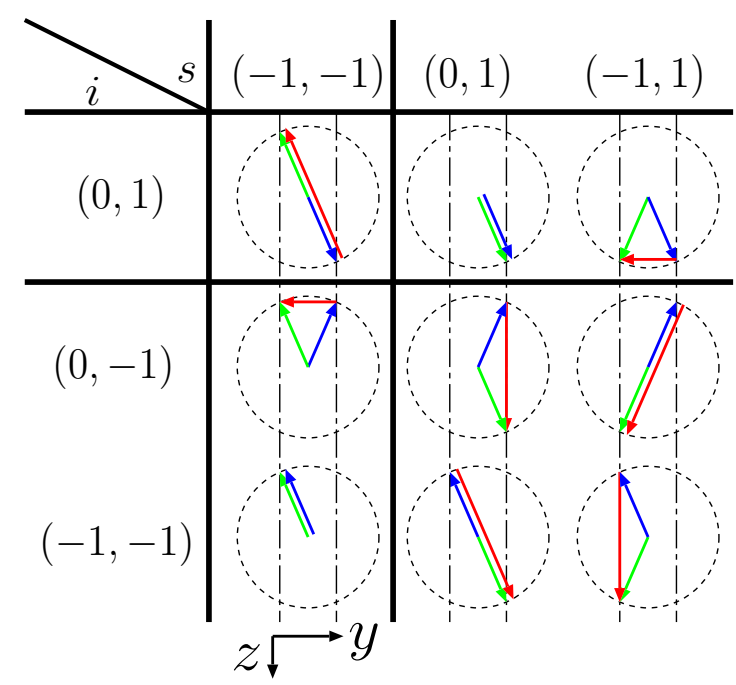

FIG. 6. The possible Brillouin scattering configurations for the sample rotated to the orientation $[\alpha, \beta]=\left[0.0^{\circ}, 33.1^{\circ}\right]$. The wave vectors of photons before the scattering $\mathbf{k}_{i}$ are shown by blue arrows. The wave vectors of the photons after the scattering $\mathbf{k}_{s}$ are shown by green arrows. The wave vectors of the absorbed phonons $\mathbf{k}_{B}$ are shown by red arrows. The $y$ and $z$ axes are shown at the bottom. In the left column the state of the photon incident on the acoustic grating is identified by the diffraction order $m_{i}$ and the direction of its propagation along the $z$ axis, $d_{i}= \pm 1$, in the form $\left(m_{i}, d_{i}\right)$. In the top row the state of the photon scattered by the acoustic grating is identified by the diffraction order $m_{s}$ and the direction of its propagation along the $z$ axis, $d_{s}= \pm 1$, in the form $\left(m_{s}, d_{s}\right)$. The distance between the dash-dotted vertical lines is equal to the wave number of the gratings $q$. The vertical and horizontal solid lines indicate the distinction of forward and backward scattering processes: The top left and bottom right processes involve the backward scattering, whereas the top right and bottom left processes involve the forward scattering. The configurations $(0,1) \rightarrow(0,1)$ and $(-1,-1) \rightarrow(-1,-1)$ are, in fact, not the scattering, but just the light passing through the sample.

by the acoustic grating, and finally reflected or diffracted by the metallic grating again. The acoustic wave involved in this peak has $\left|k_{B y}\right|=q$ and thus can be generated as described in Sec. II.

The shear Brillouin peaks expected around $9.93 \mathrm{GHz}$ $[(0,-1) \rightarrow(-1,-1)$ and $(0,1) \rightarrow(-1,1)]$ are not observed. These acoustic waves are supposed to be detected in the forward Brillouin scattering (as the index $d$ does not change sign in the process); thus the expected propagating direction for these waves is almost parallel to the surface. We have estimated that the shear acoustic phonons contributing to the Brillouin scattering processes for $[\alpha, \beta]=\left[36.4^{\circ}, 40.0^{\circ}\right]$ and $\left[26.0^{\circ}, 40.0^{\circ}\right]$ propagate at about $1^{\circ}$ and $1.5^{\circ}$ inclination angles relative to the interface, respectively. Thus, these waves at around $9.93-\mathrm{GHz}$ frequency are almost surface-skimming shear acoustic waves. The shear stresses at the interface of Al rods and glass substrate resulting from the pump laser excitation exhibit the forces along their interface. This means that the driving force is almost parallel to the propagation direction of the shear acoustic phonons that could be probed in the considered processes, and so it is almost normal to 
the required direction of the mechanical displacement in these modes. Therefore, the generation efficiency of the shear acoustic waves around $9.93 \mathrm{GHz}$ should be low. It could be, thus, expected that the amplitude of the discussed peak is importantly lower than that of the peaks experimentally observed around $25 \mathrm{GHz}$. Taking into account that in Fig. 5 the parts below $11 \mathrm{GHz}$ are diminished 5 times in amplitude for convenience, it becomes clear why the peaks predicted for surface-skimming shear acoustic waves are not distinguishable in the vicinity of the Rayleigh wave peak (which will be discussed later).

The low efficiency in the emission of the shear acoustic waves at large angles to the interface normal could be at least partially attributed to the fact that nearly skimming shear acoustic waves could be mode converted only from the evanescent modes and not from the propagating compression/dilatation modes incident on the $\mathrm{Al} / \mathrm{SiO}_{2}$ interface [26]. In other words, the shear waves propagating at small angles to the interface, i.e., nearly skimming shear acoustic waves, cannot be excited synchronously because the realvalued projections of their wave vectors on the interface are always larger than the maximal projections of the propagating longitudinal waves. Note that the shear wave in $\mathrm{SiO}_{2}$ is slower than the compression/dilatation waves both in $\mathrm{SiO}_{2}$ and in Al. This could importantly diminish the contribution to the launched shear waves of the first two from the three channels for the shear acoustic wave emission in the substrate, which were listed in Sec. II. These channels involve longitudinal to shear acoustic wave mode conversion either in transmission through or in reflection from the $\mathrm{Al} / \mathrm{SiO}_{2}$ interface. The third channel of the shear acoustic wave emission into the substrate is also importantly suppressed for the nearly surface skimming shear waves. This takes place because the synchronous transmission of the incident shear wave into the surface-skimming one without mode conversion requires the incidence on the $\mathrm{Al} / \mathrm{SiO}_{2}$ from the $\mathrm{Al}$ side of the shear acoustic waves at such angles of incidence that these incident shear waves themselves could be excited only by the mode conversion from the evanescent compression/dilatation waves, i.e., inefficiently. To summarize, the inefficient excitation of the surface-skimming shear waves can also be explained from the view point of the acoustic wave transmission or reflection at the interfaces.

The theoretically expected peaks for the shear waves in the region from 22 to $24 \mathrm{GHz}[(1,-1) \rightarrow(1,1)$ and $(0,-1) \rightarrow$ $(0,1)]$ are experimentally undetected as well. The shear acoustic waves that could contribute to these processes should not diffract the incident probe light (as $m_{s}$ is equal to $m_{i}$ ). This means that the expected acoustic waves should propagate perpendicularly to the surface. As explained in Sec. II, however, the generation of the shear acoustic waves propagating perpendicularly to the surface is inhibited by the symmetry of the grating structure, and thus, the suggested processes are theoretically impossible and experimentally undetectable.

The experimentally obtained peak around $9.0 \mathrm{GHz}$ appears below the lowest calculated Brillouin frequency of $9.93 \mathrm{GHz}$ marked in Fig. 5 for the surface-skimming shear acoustic waves. Moreover, this peak also appears for $\alpha=0$ when the detection of the shear waves is theoretically impossible. These observations indicate that the acoustic mode produc- ing this frequency peak under consideration should contain compression/dilatation acoustic field components that are detectable in the $\alpha=0$ experimental configuration. The mode exhibiting this property and propagating at a velocity lower than and close to the velocity of shear waves is known to be the surface Rayleigh acoustic wave on a mechanically free surface of an elastically isotropic half-space [32-34]. In our sample the analog of this mode is a generalized surface Rayleigh mode that accounts for the surface loading by the metallic grating. It still contains the components of the compression/dilatation acoustic field and propagates at a velocity a bit lower than the shear acoustic velocity. So we attribute the experimentally detected frequency peak around 9.0 GHz to the forward Brillouin scattering of probe light by the generalized Rayleigh wave.

Our hypothesis is supported by an approximate evaluation of the Rayleigh wave velocity in $\mathrm{SiO}_{2}$ using the velocities of the compression/dilatation and shear acoustic modes determined in our experiment. For this purpose, we first evaluate the Poisson ratio $\left[v=(1 / 2)\left(v_{l}^{2}-2 v_{t}^{2}\right) /\left(v_{l}^{2}-v_{t}^{2}\right)=\right.$ 0.17 and then use the approximate solution for the Rayleigh velocity under the assumption of its small deviation from the velocity of shear waves $\left[v_{R}=(0.875+1.125 v) v_{t} /(1+v)=\right.$ $0.91 v_{t}$ ]. This estimate predicts a peak due to probe light scattering by a surface acoustic wave with a wavelength of $380 \mathrm{~nm}$ at $9.0 \mathrm{GHz}$, which is in excellent agreement with the frequency of the experimentally observed peak. Note that the velocity of the generalized Rayleigh wave in our sample appears to be negligibly different from the velocity of the Rayleigh wave because of close values of the acoustic impedances of the $\mathrm{SiO}_{2}$ substrate and those of the $\mathrm{Al}$ rods for both the compression/dilatation and shear waves, leading to weak reflections of the acoustic waves at the $\mathrm{Al} / \mathrm{SiO}_{2}$ interface, and of small thickness of the $\mathrm{Al}$ rods in comparison with the surface acoustic wavelength, i.e., in comparison with the grating period. In our structure the thickness of the rods is nearly an order of magnitude smaller than the grating period.

Our experimental results confirm our expectations that manipulating shear acoustic waves with the assistance of metallic gratings is more complicated than manipulating compression/dilatation acoustic waves. First, the number of detectable expected Brillouin frequencies due to the shear acoustic waves is smaller than that due to the longitudinal acoustic waves because our designed metallic grating cannot launch coherent plane homogeneous shear acoustic waves in the substrate, whereas the plane homogeneous longitudinal wave is launched. This prevents monitoring with the shear waves a number of Brillouin scattering configurations, evaluated earlier with the assistance of grating by longitudinal waves in Refs. [24,25], where the acoustic wave is only reflecting and not diffracting the probe light. In the future, this discrepancy between the excitation of longitudinal and shear waves with the use of metallic gratings could be eliminated when using gratings with broken symmetry. For example, the required asymmetry could be introduced by replacing rods with a rectangular cross section by those with a trapezoidal or triangular cross section with the inclined, relative to the $\mathrm{Al} / \mathrm{SiO}_{2}$ interface, mechanically free surface.

The requirement of a nonzero $\alpha$ angle for the shear acoustic wave detection (see Sec. II) decreases the number of 
diffraction orders where the probe light could propagate in the sample. For the propagation of both incident and scattered light, i.e., to avoid the evanescent light fields, the following inequality should be fulfilled [see Eq. (7)]:

$$
-\sqrt{n^{2} k^{2}-k_{x}^{2}}-k_{y}<m_{i, s} q<\sqrt{n^{2} k^{2}-k_{x}^{2}}-k_{y} .
$$

Thus, the requirement of nonzero $k_{x}$ for the detection of the coherent shear acoustic waves by the time-domain Brillouin scattering could lead to narrowing of the interval for the allowed values of $m_{i}$ and $m_{s}$. If required for the observation of the higher-order diffraction processes [24], this narrowing due to nonzero $\alpha$ could be compensated by increasing the grating period, i.e., by diminishing $q$. However, it should not be forgotten that, theoretically, in the grating with the ratio of the rod width to the grating period precisely equal to $50 \%$ diffraction in the even orders is prohibited. So for efficiently multiplexing the acoustic and optical beams via higher diffraction orders (for example, by the second one) the ratio of the rod width to the grating period should be adjusted.

Another interesting extension of this work would be the use of two-dimensional periodic metallic structures. In this case, the probe light is diffracted not only within a single plane but also in the directions specified by the two-dimensional reciprocal lattice vector of the periodic structure. This could allow us to fulfill the requirements for the shear acoustic wave detection even with the probe light in simpler optical configurations, such as normal incidence and normal reflection from the sample.

It has been pointed out that the grating technique for the compression/dilatation acoustic waves could be used to explore the dispersion of the acoustic waves [24]. This also would be possible for the shear acoustic waves. For this purpose, we need to increase the number of accessible Brillouin peaks (currently, we see only a single peak for the shear waves) as discussed above, i.e., via use of the higher-order acoustic diffraction, use of the broken-symmetry grating structures, use of several different angles of probe light incidence, etc.

\section{CONCLUSIONS}

We have experimentally evidenced the generation and detection of $\mathrm{GHz}$ shear acoustic waves by the time-resolved Brillouin scattering measurements for a transparent sample coated with a grating-type metallic optoacoustic transducer. For detection, it is required to have the probe light wave vector nonparallel to the plane perpendicular to the grating rods. The peak frequencies (Brillouin frequencies) in the spectrum of the transient reflectivity agree well with the theoretically predicted values. The absence of several calculated peaks for shear acoustic waves in the experimentally obtained spectra is also theoretically explained. The obtained results open up a way to utilize the time-domain Brillouin scattering to monitor simultaneously compression/dilatation and shear acoustic waves at several frequencies in order to obtain complete information on the acoustical and optical parameters of the materials. New perspectives for time-domain Brillouin scattering imaging of inhomogeneous materials with shear and compression/dilatation acoustic waves could be envisioned as well.

\section{ACKNOWLEDGMENTS}

O.M. is partially supported by the Acoustic HUB of Région des Pays de la Loire in France, by a Grant-in-Aid for Scientific Research from the Japan Society for the Promotion of Science, and by a research grant from Insight K.K. We would like to thank the Open Facility (Hokkaido University Sousei Hall) for sample fabrication.
[1] C. Thomsen, H. T. Grahn, H. J. Maris, and J. Tauc, Phys. Rev. B 34, 4129 (1986).

[2] H. T. Grahn, H. J. Maris, and J. Tauc, IEEE J. Quantum Electron. 25, 2562 (1989).

[3] O. B. Wright, J. Appl. Phys. 71, 1617 (1992).

[4] A. Devos, R. Côte, G. Caruyer, and A. Lefévre, Appl. Phys. Lett. 86, 211903 (2005).

[5] R. Côte and A. Devos, Rev. Sci. Instrum. 76, 053906 (2005).

[6] P.-A. Mante, Y.-R. Huang, S.-C. Yang, T.-M. Liu, A. A. Maznev, J.-K. Sheu, and C.-K. Sun, Ultrasonics 56, 52 (2015).

[7] O. Matsuda, M. C. Larciprete, R. L. Voti, and O. B. Wright, Ultrasonics 56, 3 (2015).

[8] V. E. Gusev and P. Ruello, Appl. Phys. Rev. 5, 031101 (2018).

[9] D. H. Hurley, O. B. Wright, O. Matsuda, V. E. Gusev, and O. V. Kolosov, Ultrasonics 38, 470 (2000).

[10] T. Bienville and B. Perrin, in Proceedings of the World Congress on Ultrasonics: WCU 2003, Paris, France, 7-10 September 2003 (Société française d'acoustique, Paris, 2003), pp. 813-816.

[11] O. Matsuda, O. B. Wright, D. H. Hurley, V. E. Gusev, and K. Shimizu, Phys. Rev. Lett. 93, 095501 (2004).

[12] T. Pezeril, V. Gusev, D. Mounier, N. Chigarev, and P. Ruello, J. Phys. D 38, 1421 (2005).
[13] T. Pezeril, N. Chigarev, P. Ruello, S. Gougeon, D. Mounier, J.-M. Breteau, P. Picart, and V. Gusev, Phys. Rev. B 73, 132301 (2006).

[14] R. N. Kini, A. J. Kent, N. M. Stanton, and M. Henini, Appl. Phys. Lett. 88, 134112 (2006).

[15] O. Matsuda, O. B. Wright, D. H. Hurley, V. Gusev, and K. Shimizu, Phys. Rev. B 77, 224110 (2008).

[16] T. Pezeril, C. Klieber, S. Andrieu, and K. A. Nelson, Phys. Rev. Lett. 102, 107402 (2009).

[17] M. Lejman, G. Vaudel, I. C. Infante, V. E. Gusev, B. Dkhil, and P. Ruello, Nat. Commun. 5, 4301 (2014).

[18] T. Pezeril, Opt. Laser Technol. 83, 177 (2016).

[19] M. Lejman, G. Vaudel, I. C. Infante, I. Chaban, T. Pezeril, M. Edely, G. F. Nataf, M. Guennou, J. Kreisel, V. E. Gusev et al., Nat. Commun. 7, 12345 (2016).

[20] C. Rossignol, J. M. Rampnoux, M. Perton, B. Audoin, and S. Dilhaire, Phys. Rev. Lett. 94, 166106 (2005).

[21] T. Dehoux, N. Chigarev, C. Rossignol, and B. Audoin, Phys. Rev. B 76, 024311 (2007).

[22] M. Kouyate, T. Pezeril, D. Mounier, and V. Gusev, J. Appl. Phys. 110, 123526 (2011).

[23] V. Gusev, Appl. Phys. Lett. 94, 164105 (2009). 
[24] O. Matsuda, T. Pezeril, I. Chaban, K. Fujita, and V. Gusev, Phys. Rev. B 97, 064301 (2018).

[25] H. N. Lin, H. J. Maris, L. B. Freund, K. Y. Lee, H. Luhn, and D. P. Kem, J. Appl. Phys. 73, 37 (1993).

[26] S. M. Nikitin, V. Tournat, N. Chigarev, A. Bulou, B. Castagnede, A. Zerr, and V. Gusev, J. Appl. Phys. 115, 044902 (2014).

[27] Note that the grating shape and profile along the propagation direction cannot be maintained as it propagates since the phase velocity along the $z$ direction of the acoustic waves composing the grating depends on the frequency and thus is dispersive [23].

[28] CRC Handbook of Chemistry and Physics, 85th ed., edited by D. R. Lide (CRC Press, Boca Raton, FL, 2004).
[29] M. Grossmann, M. Schubert, C. He, D. Brick, E. Scheer, M. Hettich, V. Gusev, and T. Dekorsy, New J. Phys. 19, 053019 (2017).

[30] M. A. Ghanem, T. Dehoux, L. Liu, G. L. Saux, L. Plawinski, M.-C. Durrieu, and B. Audoin, Rev. Sci. Instrum. 89, 014901 (2018).

[31] J. D. G. Greener, E. de Lima Savi, A. V. Akimov, S. Raetz, Z. Kudrynskyi, Z. D. Kovalyuk, N. Chigarev, A. Kent, A. Patane, and V. Gusev, ACS Nano 13, 11530 (2019).

[32] I. A. Victorov, Rayleigh and Lamb Waves (Plenum, New York, 1967).

[33] J. D. Achenbach, Wave Propagation in Elastic Solids (NorthHolland, Amsterdam, 1973).

[34] B. A. Auld, Acoustic Fields and Waves in Solids, 2nd ed. (Krieger, Malabar, FL, 1990), Vol. 2. 